\title{
Revisiting liquid lubrication methods by means of a fully coupled approach combining plastic deformation and liquid lubrication
}

\author{
Üstünyagiz, Esmeray; Christiansen, Peter; Nielsen, Chris Valentin; Bay, Niels Oluf; Martins, Paulo AF
}

Published in:

Proceedings of the Institution of Mechanical Engineers, Part J: Journal of Engineering Tribology

Link to article, DOI:

$10.1177 / 1350650117697825$

Publication date:

2017

Document Version

Peer reviewed version

Link back to DTU Orbit

Citation (APA):

Üstünyagiz, E., Christiansen, P., Nielsen, C. V., Bay, N. O., \& Martins, P. AF. (2017). Revisiting liquid lubrication methods by means of a fully coupled approach combining plastic deformation and liquid lubrication. Proceedings of the Institution of Mechanical Engineers, Part J: Journal of Engineering Tribology, 231(11), 1425-1433. https://doi.org/10.1177/1350650117697825

\section{General rights}

Copyright and moral rights for the publications made accessible in the public portal are retained by the authors and/or other copyright owners and it is a condition of accessing publications that users recognise and abide by the legal requirements associated with these rights.

- Users may download and print one copy of any publication from the public portal for the purpose of private study or research.

- You may not further distribute the material or use it for any profit-making activity or commercial gain

- You may freely distribute the URL identifying the publication in the public portal 


\title{
Revisiting liquid lubrication methods by means of a fully- coupled approach combining plastic deformation and liquid lubrication
}

\author{
Esmeray Üstünyagiz ${ }^{1}$, Peter Christiansen ${ }^{1}$, Chris V Nielsen $^{1}$, Niels Bay ${ }^{1}$ and Paulo AF Martins ${ }^{2}$ \\ ${ }^{1}$ Department of Mechanical Engineering, Technical University of Denmark, Denmark \\ ${ }^{2}$ IDMEC, Instituto Superior Tecnico, Universidade de Lisboa, Portugal
}

\begin{abstract}
This paper presents a new approach based on a fully coupled procedure in which the lubricant flow and the plastic deformation of the metallic material in metal forming are solved simultaneously. The proposed method is an alternative to conventional modelling techniques which allow studying the effect of a broad range of parameters directly on the friction conditions. The approach is applied to strip reduction of a sheet with mesoscopic surface pockets in order to investigate the escape of lubricant from the pocket by means of Micro Plasto HydroDynamic Lubrication (MPHDL) and Micro Plasto HydroStatic Lubrication (MPHSL). For the investigation on MPHSL the friction along the tool-workpiece contact interface and the back tension are taken as parameters, and the backward escape (MPHDL) is investigated by variations in lubricant viscosity by means of a combined numerical and analytical model, and by variations in drawing speed. Good agreement is found with experimental observations.
\end{abstract}

\section{Keywords}

Plastic deformation, viscous flow, finite element method, liquid lubrication mechanisms, lubricant pockets

\section{Introduction}

Liquid lubrication of metal forming processes is characterized as the mixed lubrication regime, where the interface pressure is carried partly by the pressurized lubricant trapped in surface pockets and partly by 
asperity contact with a possible boundary film between the tool and the workpiece. These conditions are known to have a considerable influence on friction, wear and resulting surface topography.

Early studies of the influence of entrapped lubricant on real contact area between tool and workpiece in metal forming were carried out by Kudo ${ }^{1}$ and Nellemann et al. ${ }^{2}$ during the 1960's and 1970's. In the early 1980's Mizuno and Okamato ${ }^{3}$ proposed the escape of entrapped lubricant from pockets in order to better understand the interaction between tool and workpiece. This mechanism was supported by Kudo et al. ${ }^{4}$ and experimentally confirmed by Azushima et al. $^{5-6}$, who performed strip reduction tests through a wedge shaped, transparent die and observed lubricant entrapment, pressurization and subsequent escape from mesoscopic pockets in the strip directly. Figure 1(a) shows a schematic representation of the Micro Plasto HydroStatic Lubrication (MPHSL) in which the forward escape of lubricant occurs when the lubricant pressure $p_{\text {liq }}$ reaches the sealing pressure $p_{f}$ at the front end of the pocket. Alternatively, Figure 1(b) presents the Micro Plasto HydroDynamic Lubrication (MPHDL) mechanism in which the backward escape occurs when the viscous drag of the lubricant creates a liquid pressure $p_{\text {liq }}$ at the rear end of the pocket that reaches the sealing pressure $p_{r}$ at the back of the pocket.

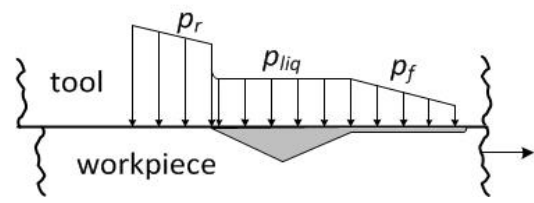

(a)

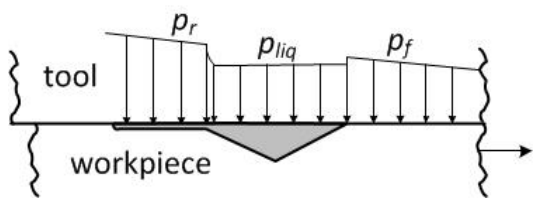

(b)

Figure 1. Schematic representation of the two mechanisms of lubricant escape. (a) Micro Plasto HydroStatic Lubrication; (b) Micro Plasto HydroDynamic Lubrication.

Bech et al. ${ }^{7}$ and Shimizu et al. ${ }^{8}$ used an experimental set-up similar to Azushima et al. ${ }^{5-6}$ in order to investigate the influence of pocket geometry, drawing speed and lubricant viscosity, among other parameters, on the escape of entrapped lubricant. They also established an analytical framework capable of modelling the influence of these parameters on the pressure build-up and lubricant escape. A similar work was performed by Lo and Wilson ${ }^{9}$. More recently, Azushima et al. ${ }^{10}$ studied pressure build-up and lubricant escape from a central pocket in upsetting by means of a rigid plastic finite element analysis. 
In the last decades, there have not been significant advances in the modelling of contact with friction between workpiece and tools. The common practice is still to treat friction as a traction boundary condition resulting from the application of shear stresses along the interface between workpiece and tool and to assume shear stresses to follow either Coulomb's law or the law of constant friction stress.

This means that a broad range of parameters, which are known to influence the frictional conditions in metal forming with liquid lubrication, such as surface expansion, sliding length, sliding speed, lubricant viscosity, and geometry and surface topography of tools and workpiece, are hidden in the tuning of friction and not directly taken into consideration. Thus, there is room for improving the current state-of-the-art in friction modelling by including some of the above mentioned parameters in friction modelling.

This was recently recognized by Carretta et al. ${ }^{11}$ who proposed a combination of computational fluid dynamics and solid mechanics to analyse micro-plasto-hydrodynamic effects in the numerical simulation of strip reduction. In their work, they identified numerical difficulties caused by the differences in stiffness of metals and fluids, which may be up to 9 or 10 orders of magnitude, and by excessive mesh distortion of the fluid. The latter was claimed to be solved by employing an Arbitrary Lagrangian-Eulerian (ALE) formulation, in which the motion of the fluid mesh is uncoupled from the general motion of the workpiece mesh. However, it is worth mentioning that coupling of computational fluid dynamics and solid mechanics is generally not straightforward, because typical implementations of the Navier-Stokes equations work with velocities and pressures, whereas commonly utilized formulations of solid mechanics work with displacements.

This paper deals with an alternative approach for the finite element modelling of friction in metal forming based on the finite element flow formulation ${ }^{12-13}$. The main idea is to utilize a thin interface layer of elements at the contact interface between the workpiece and tool, like it was originally proposed by Hartley et al. ${ }^{14}$ in the late 1970 's. But, instead of treating the interface layer as solid elements with a fictitious small stiffness as performed by Hartley et al. ${ }^{14}$, the line of attack of this paper is to treat the interface layer as a viscous fluid.

A computer program that allows treating metals as non-Newtonian, high viscous, incompressible fluids and lubricants at the thin interface layer as viscous incompressible (or nearly incompressible) Newtonian fluids was developed by the authors for the above mentioned purpose $\mathrm{e}^{15}$. This work was carried out on top of the 
in-house computer program i-form that has been extensively validated against experimental measurements of metal forming processes since the end of the $1980{ }^{\prime}{ }^{13}$. The reason for choosing the finite element flow formulation is its intrinsic velocity-pressure characteristics, which allows performing a stronger numerical coupling between metal and fluid than that achieved by combining typical formulations used in computational fluid dynamics and solid mechanics.

The presentation is focused on the application of the new proposed computational approach to the investigation of pressurization and escape of entrapped lubricant by MPHDL and MPHSL in the strip reduction test. The forward escape (MPHSL), which is analysed numerically, investigates the influence of back tension and friction along the tool-workpiece interface. The backward escape (MPHDL) is analysed by a combined numerical and analytical procedure that investigates the influence of shear viscosity of the lubricant and drawing speed. Numerical predictions are compared with the experimental results of Bech et al. ${ }^{7}$.

\section{Theoretical background}

The differential equation of linear momentum resulting from force equilibrium in a fluid particle is given by,

$\frac{\partial \sigma_{i j}^{\prime}}{\partial x_{j}}-\frac{\partial p}{\partial x_{i}}+\rho g_{i}=\rho\left(\frac{d u_{i}}{d t}+u_{j} \frac{\partial u_{i}}{\partial x_{j}}\right)$

The left-hand side of equation (1) is related to viscous forces, pressure and body forces. The symbol $p$ denotes the hydrostatic pressure and $\sigma_{i j}^{\prime}$ represents the deviatoric (viscous) stresses, which are related to the total stresses $\sigma_{i j}$ as follows, $\sigma_{i j}=\sigma_{i j}^{\prime}-p$. The symbols $\rho$ and $g_{i}$ designate density and gravity acceleration, respectively.

The right-hand side of equation (1) is related to inertia effects. If convective terms are neglected, due to small space-dependency, this term reduces to, 
$\rho \frac{d u_{i}}{d t}=\rho a_{i}$

where $a_{i}$ is the acceleration.

Because deviatoric (viscous) stresses $\sigma_{i j}^{\prime}$ in fluids are proportional to strain rates $\dot{\varepsilon}_{i j}$,

$\sigma_{i j}^{\prime}=2 \mu_{S} \dot{\varepsilon}_{i j}$

by replacing equations (2) and (3) into equation (1), the differential equation of linear momentum for incompressible fluid flow with constant viscosity reduces to the so-called Navier-Stokes equation,

$\mu_{S} \frac{\partial^{2} u_{i}}{\partial x_{j}^{2}}-\frac{\partial p}{\partial x_{i}}+\rho g_{i}=\rho \frac{d u_{i}}{d t}$

where $\mu_{S}$ is the shear viscosity, which takes constant values $\mu_{S}=\mu_{0, S}$ for Newtonian fluids and strain rate dependent values $\mu_{S}=\mu_{S}\left(\dot{\varepsilon}_{i j}\right)$ for non-Newtonian fluids (e.g. metals).

The irreducible finite element flow formulation that will be utilized for modelling plastic deformation and liquid lubrication is derived from the discretization of the weak form of equation (4) by means of finite elements, after neglecting the inertia effects due to small loading rates in metal forming,

$\int_{V} \sigma_{i j}^{\prime} \delta \dot{\varepsilon}_{i j}^{\prime} d V+K \int_{V} \dot{\varepsilon}_{i i} \delta \dot{\varepsilon}_{i i} d V-\int_{S_{T}} t_{i} \delta u_{i} d S+\int_{V} \rho g_{i} \delta u_{i} d V=0$

As seen in equation (5), the separation between deviatoric (viscous) and volumetric terms is similar to equation (1) and enables a direct correlation of shear and bulk viscosity for metals and fluids undergoing laminar flow. For example, the deviatoric stresses of metals may be seen as the stress response of nonNewtonian fluids of very high viscosity $\mu_{S}=\bar{\sigma} / 3 \dot{\bar{\varepsilon}}$ (in close analogy to equation (3)),

$\sigma_{i j}^{\prime}=\frac{2}{3} \frac{\bar{\sigma}}{\dot{\bar{\varepsilon}}} \dot{\varepsilon}_{i j}^{\prime}$ 
Conversely, the penalty factor $K$, utilized to ensure the incompressibility of metals may be seen as the bulk viscosity $\mu_{V}$ of fluids relating the changes $\dot{\varepsilon}_{i i}$ in volume $V$ with the hydrostatic pressure $p$ (or, average stress $\sigma_{m}$ ),

$K=\mu_{V}$ with $\sigma_{m}=-p=K \dot{\varepsilon}_{i i}$

As a result of the above equations, it may be concluded that the velocity-pressure $(u-p)$ characteristics of the flow formulation leads to a natural coupling between metals and fluids, in which metals are treated as incompressible fluids of very high viscosity (equation (6)). Thus, one of the main challenges in coupling of conventional finite element programs used in fluid dynamics and solid mechanics can be straightforwardly solved by means of the new proposed approach based on the flow formulation due to its capability of solving velocities $u_{i}$ and hydrostatic pressures $p$ simultaneously.

Moreover, the implementation of the proposed approach in existing flow formulation based finite element computer programs such as $\mathrm{i}$-form ${ }^{13}$ is easy to be carried out as it mainly requires replacing the $\mathbf{D}$ matrix built upon the Levy-Mises constitutive equations by the following $\mathbf{D}_{\mu}$ matrix resulting from equation (3),

$\mathbf{D}=\overline{\bar{\sigma}}\left[\begin{array}{cccccc}\dot{\bar{\varepsilon}} & 0 & 0 & 0 & 0 & 0 \\ 0 & 2 / 3 & 0 & 0 & 0 & 0 \\ 0 & 0 & 2 / 3 & 0 & 0 & 0 \\ 0 & 0 & 0 & 1 / 3 & 0 & 0 \\ 0 & 0 & 0 & 0 & 1 / 3 & 0 \\ 0 & 0 & 0 & 0 & 0 & 1 / 3\end{array}\right] \quad \mathbf{D}_{\mu}=\mu_{S}\left[\begin{array}{cccccc}2 & 0 & 0 & 0 & 0 & 0 \\ 0 & 2 & 0 & 0 & 0 & 0 \\ 0 & 0 & 2 & 0 & 0 & 0 \\ 0 & 0 & 0 & 1 & 0 & 0 \\ 0 & 0 & 0 & 0 & 1 & 0 \\ 0 & 0 & 0 & 0 & 0 & 1\end{array}\right]$

As a result of this, the stiffness matrix of the interface layer of fluid elements that are utilized to discretize the lubricants is calculated from $\mathbf{K}_{\mu}=\mathbf{B}^{T} \mathbf{D}_{\mu} \mathbf{B}$, where $\mathbf{B}$ is the classical strain rate matrix.

\section{Methods and procedures}

\section{Experimental work}


The aim of the paper is to present a fully coupled approach in which the lubricant flow and the plastic deformation of the metallic material are solved simultaneously. Therefore, as with all new numerical developments, it is necessary to investigate its accuracy, reliability and validity. This was carried out by assessing numerical predictions by experimental data taken from a previous paper published by one of the authors (Bech et al. ${ }^{7}$ ). Experimental data on the escape of entrapped lubricant was obtained for the drawing of an aluminum AA5052 strip of $2 \mathrm{~mm}$ thickness through a wedge shaped die with die angle $3^{\circ}$ and reduction in thickness equal to $20 \%$. The upper side of the strip was provided with pyramidal shaped pockets (1x1 mm base lengths and $10^{\circ}$ slope), which were filled with lubricant prior to drawing. A transparent, hardened, glass die allowed observation of the lubrication mechanisms on the strip directly (Figure 2), In this way the influence of friction, back tension, lubricant viscosity and drawing speed on forward (MPHSL) and backward (MPHDL) lubricant escape were studied.

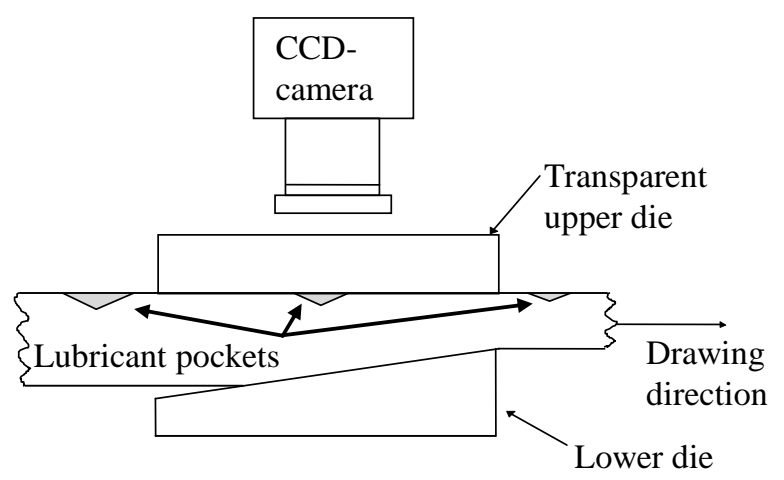

Figure 2. Outline of the experimental set-up utilized by Bech et al. ${ }^{7}$.

The simulation conditions that were utilized to validate the new proposed approach for modelling plastic deformation and liquid lubrication are shown in Table 1. The values in underline bold are taken as reference while the others are variants of a particular parameter keeping all the others at their reference values.

As shown in the schematic drawings included in Table 1, the increase in lower die friction and back tension leads to a shift from backward towards forward lubricant escape, whereas the increase in viscosity or velocity gives rise to an opposite effect. 
Table 1. Summary of the experimental investigation of lubricant escape from pockets in strip surface performed by Bech et al. .

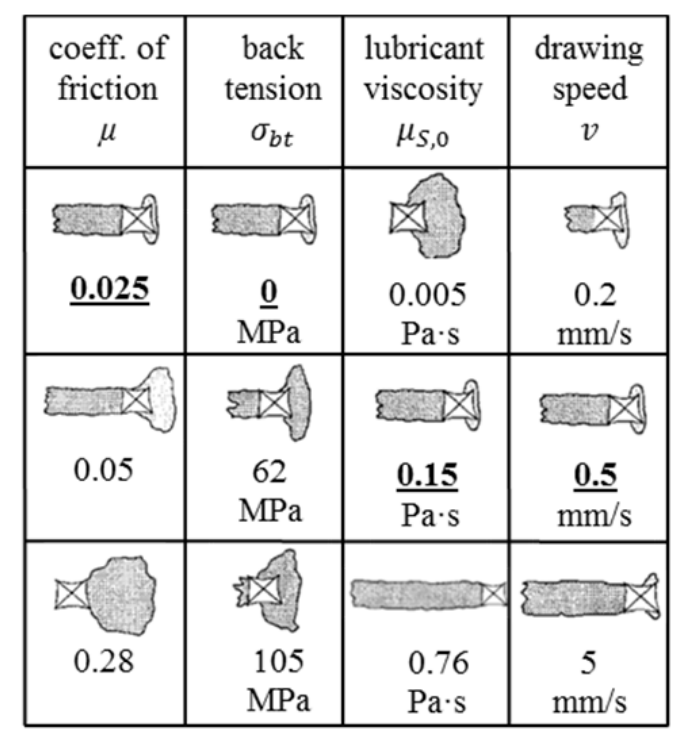

The following section presents and discusses results from a parametric study showing that increased values of friction along the lower die and back tension promote forward lubricant escape, while increased values of lubricant viscosity and drawing speed promote backward lubricant escape.

\section{Numerical modelling}

The stress-strain curve of the AA5052 strip was approximated by the following Ludwik-Hollomon equation ${ }^{7}$,

$\bar{\sigma}=321 \bar{\varepsilon}^{0.08}[\mathrm{MPa}]$

The main process parameters utilized in numerical modelling were taken from Bech et al. ${ }^{7}$. However, there was a need to make additional assumptions due to lack of information. For example, authors used (i) sticking friction conditions along the contact interfaces between the strip and the lubricant in order to replicate the absence of relative velocity, (ii) a constant friction factor $m=0.3$ along the contact of the strip with the upper die made from hardened glass and (iii) a bulk viscosity of the lubricant $\mu_{V}=1500 \mathrm{MPa} \cdot \mathrm{s}$. 
The plastic deformation of the strip was modelled in two-dimensions under plane strain deformation, which means that the pyramidal pockets were considered as infinitely long grooves in the sheet perpendicular to the drawing direction. It should be kept in mind for the following sections that replacing the pyramidal pockets by infinitely long grooves may promote deformation of the pocket due to reduced stiffness. This may lead to an increase of the hydrodynamic pressure and hence affect the lubricant escape.

The sheet and lubricant were discretized by means of linear quadrilateral elements whereas the dies were treated as rigid bodies and discretized by means of linear contact elements (Figure 3(a)). The large difference in stiffness between metals and lubricants commonly gives rise to unacceptable mesh distortion inside the pockets as soon as the strip material starts to be drawn. Because this problem leads to numerical difficulties, the authors developed a continuous smoothing algorithm that can be easily and effectively applied to the lubricant mesh without influencing the quality of the results due to the history independence of its constitutive equations (refer to equation(3)). Referring to Figure 3(b) the smoothing algorithm is based on fixing the vertices $V_{1}, V_{2}$ and $V_{3}$ identified in Figure 3(b) by the boundary nodes where the neighboring edge segments have a mutual angle greater than a critical angle. The overall quality of the mesh is improved by smoothing all other nodes. First, the edges between vertices $E_{1}, E_{2}$ and $E_{3}$ are defined. A parametric based procedure similar to the one developed by the authors ${ }^{16}$ is utilized for the edge smoothing. The present algorithm is improved by handling the smoothing in one step by uniform distribution of the edge nodes along the (in general curved) edge. The full points in Figure 3(b) represent the position of nodes lying on the edge before smoothing whereas the dotted circles illustrates the nodal positions after smoothing. Finally, the surface $S$ is smoothened by Laplacian surface smoothing. A typical simulation consisting of a mesh with approximately 9300 nodal points and 9100 elements, similar to that shown in Figure 3(a), required approximately 4 hours to be finished in a laptop computer equipped with an Intel CPU e5-1660 (3.0 GHz) processor. The convergence norm for the velocity field $|\Delta u| /|u|$ was chosen as 0.001 . 


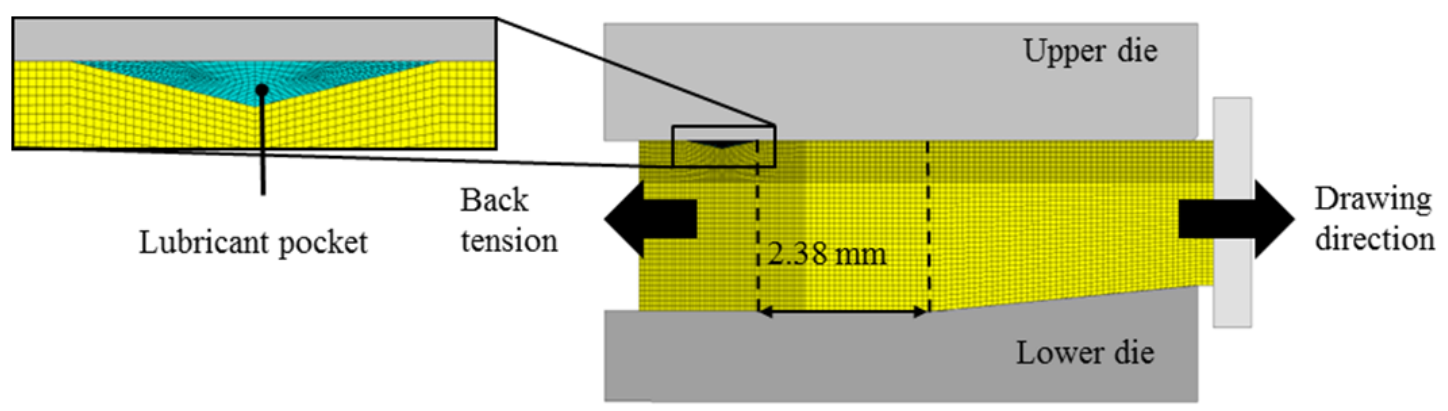

(a)

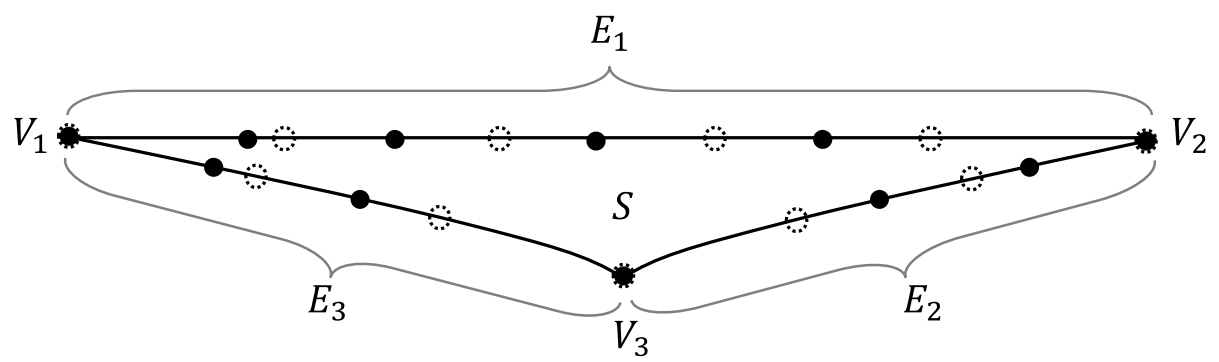

(b)

Figure 3. (a) Finite element model of strip reduction with a detail of the pocket on the strip surface. (b) Schematic representation of smoothing for the pocket geometry.

\section{Results and discussion}

\section{MPHSL - Friction along the lower die}

The influence of friction along the lower die on the forward lubricant escape was analysed by performing numerical simulations with various friction factors along the contact interface between the inclined lower die and the strip material. The simulations were performed with constant values of the back tension $\sigma_{b t}=0$ MPa, lubricant viscosity $\mu_{S, 0}=0.15 \mathrm{~Pa} \cdot \mathrm{s}$ and drawing speed $v=0.2 \mathrm{~mm} / \mathrm{s}$ in order to replicate the experimental conditions of Bech et al. ${ }^{7}$ (refer to Table 1).

Figure 4 shows the build-up of liquid pressure $p_{\text {liq }}$ and sealing pressure $p_{f}$ at the pocket front (notation according to Figure 1) as a function of the strip displacement during the drawing process. In the numerical model the pocket is initially placed $2.38 \mathrm{~mm}$ away from the entrance of the reduction zone as shown in 
Figure 3(a), while Figure 4 presents pressures after $2.8 \mathrm{~mm}$ strip displacement, where the pocket has entered the reduction zone, such that the liquid pressure buildup has started. Two different values of the friction factor were chosen in order to model frictionless $(m=0)$ and typical $(m=0.3)$ conditions of drawing.

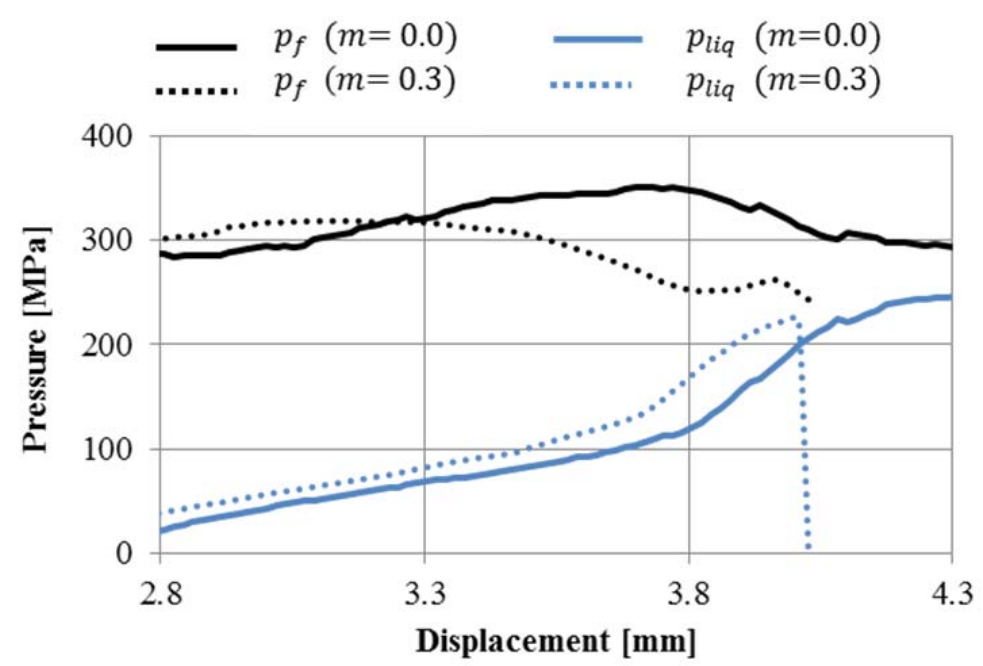

Figure 4. Evolution of the liquid lubricant pressure $p_{\text {liq }}$ and the front sealing pressure $p_{f}$ of the pocket with respect to displacement for two friction factors $m$ along the inclined lower die.

As seen in the figure, the liquid pressure $p_{\text {liq }}$ increases as the pocket is drawn through the inclined plastic deformation zone. The forward sealing pressure $p_{f}$ remains above 200 MPa due to previous plastic deformation of the strip material but its overall value depends on the friction along the lower die.

For example, in case of $m=0$ the pressure $p_{\text {liq }}$ builds up to $250 \mathrm{MPa}$ without reaching the sealing pressure $p_{f}$, which remains approximately equal to $300 \mathrm{MPa}$. This means that frictionless conditions in the lower die do not promote forward escape of lubricant, as it was experimentally observed by Bech et al. ${ }^{7}$ (refer to Table 1).

In contrast, the finite element predictions for $m=0.3$ suggest forward escape of the lubricant because the build-up of pressure $p_{\text {liq }}$ reaches the sealing pressure $p_{f}$, which is lower in this case than for $m=0$. The simulated results with increasing friction $m$ show the same tendency as the experimental results with increasing Coulomb friction $\mu$ that are provided in Table 1 and, although the finite element model does 
not replicate the flow of lubricant away from the pocket, the drop of pressure $p_{\text {liq }}$ in the numerical model can also be explained by an instantaneous change in volume. In fact, once the pressure $p_{\text {liq }}$ inside the pocket overtakes the adjacent sealing pressure $p_{f}$ there is a sudden increase in the volume of the pocket that justifies the aforementioned drop of pressure $p_{\text {liq }}$ from a numerical point of view. This phenomenon is not reported in the literature as the focus is commonly placed on the escape of lubricant from the pocket and associated drop in pressure $p_{\text {liq }}$.

Figure 5 shows the finite element predicted onset of lubricant escape for different friction factors $m$. As seen, the onset of lubricant escape occurs earlier with increasing friction on the lower die, in close agreement with the parameter study performed by Bech et al. ${ }^{7}$, which shows that an increase in friction promotes the forward escape at the expense of backward escape (see Table 1). However, this agreement can only be seen as qualitative due to the different shapes of the actual and finite element model of the pocket (pyramidal vs. infinite long groove).

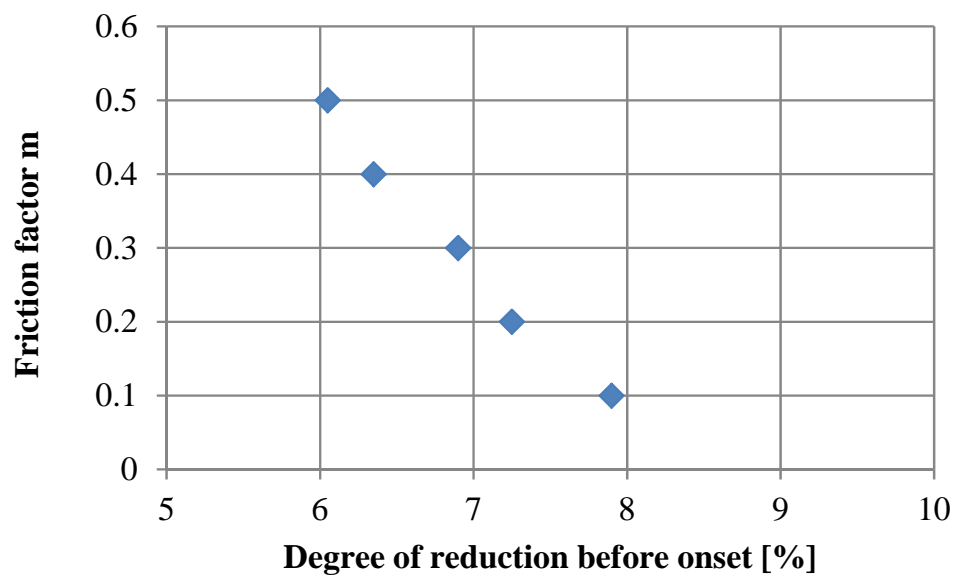

Figure 5. Dependency of the onset of lubricant escape on the friction factor at the inclined lower die.

\section{MPHSL - Back tension}

The influence of back tension on the forward lubricant escape was analysed by performing numerical simulations with various back tension $\sigma_{b t}$ values applied in the leftmost end of the strip material (Figure 
3). The simulations were performed with three different values of back tension $\sigma_{b t}=0,30,62 \mathrm{MPa}$, and constant values of friction along the lower die $m=0$, lubricant viscosity $\mu_{S, 0}=0.15 \mathrm{~Pa} \cdot \mathrm{s}$ and drawing speed $v=0.5 \mathrm{~mm} / \mathrm{s}$. These values follow the experimental conditions of Bech et al. ${ }^{7}$ that are summarized in Table 1.

Figure 6 shows that an increase in back tension $\sigma_{b t}$ will lead to a significant decrease in the sealing pressure $p_{f}$. The liquid pressure $p_{\text {liq }}$ is also found to decrease, but not to the same extent, thereby, implying an increasing tendency to lubricant forward escape, as it was experimentally observed by Bech et al. ${ }^{7}$ (Table 1).

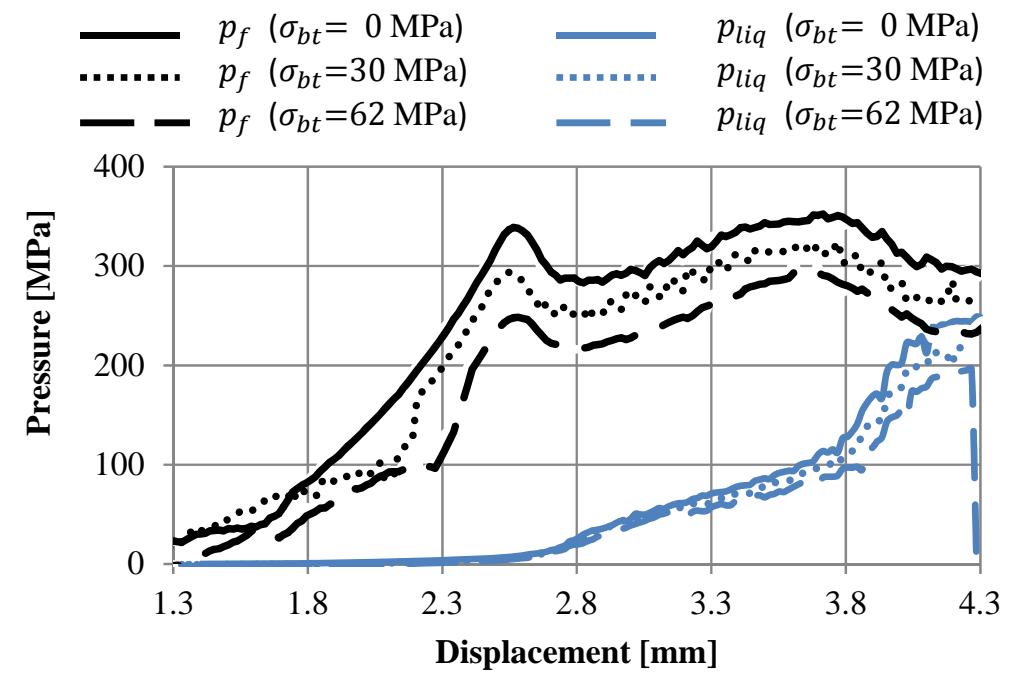

Figure 6. Evolution of liquid pressure $p_{\text {liq }}$ and the front sealing pressure $p_{f}$ of the pocket with respect to displacement for various back tension $\sigma_{b t}$ values.

\section{MPHDL - Shear viscosity}

The investigation on the backward lubricant escape was focused on the influence of shear viscosity and drawing speed. This sub-section deals with the influence of pressure dependent shear viscosity $\mu_{S}$,

$\mu_{S}=\mu_{S, 0} \exp \left(\alpha p_{\text {liq }}\right)$ 
and makes use of finite element models similar to those utilized in the previous sub-sections.

The simulations were performed with three different values of shear viscosity $\mu_{\mathrm{S}, 0}=0.005,0.76,1.5 \mathrm{~Pa} \cdot \mathrm{s}$ and a pressure-viscosity coefficient ${ }^{7} \alpha=2.1 \times 10^{-8} \mathrm{~Pa}^{-1}$. Constant values of friction along the lower die $m=0$, back tension $\sigma_{b t}=0 \mathrm{MPa}$ and drawing speed $v=0.5 \mathrm{~mm} / \mathrm{s}$ were chosen from the experimental conditions summarized in Table 1.

The numerical simulation of the lubricant escape caused by the local increase of $p_{\text {liq }}$ at the rear end of the pocket was not successfully carried out due to limited finite element discretization and unacceptable distortion of the lubricant mesh. It is worth noting that this localized mesh distortion should not be confused with the global mesh distortion of the overall lubricant mesh (pocket) that is effectively solved by the previously mentioned smoothing algorithm.

To overcome the above mentioned difficulty, it was decided to combine the finite element simulation with an analytical model of the increase in liquid pressure $p_{\text {liq }}$ at the rear end of the pocket based on the Reynolds equation ${ }^{7}$ :

$\frac{d p_{\text {liq }}}{d x}=6 \mu_{s} v \frac{h-h_{m}}{h^{3}}$

In the previous equation, $h$ is the local film thickness, $h_{m}$ is the film thickness in the plateau, $\mu_{\mathrm{S}}$ is the pressure dependent viscosity, $v$ is the sliding velocity between tool and liquid and $p_{l i q}$ is the liquid pressure.

Considering a pocket edge with a radius of curvature $R=80 \mu \mathrm{m}$ and a film thickness $h_{m}=0.1 \mu \mathrm{m}$ on top of the asperities ${ }^{7}$, the local increase of the liquid pressure $\Delta p_{\text {liq }}$ for two different shear viscosities $\mu_{s, 0}=0.76,1.5 \mathrm{~Pa} \cdot \mathrm{s}$ is equal to $26.9 \mathrm{MPa}$ and $114.3 \mathrm{MPa}$, respectively. Now, because the required amount of $\Delta p_{\text {liq }}$ for allowing the lubricant to escape had been previously estimated as $32 \mathrm{MPa}^{17}$, it may be concluded that for viscosities larger than $\mu_{S, 0}=0.76 \mathrm{~Pa} \cdot \mathrm{s}$ backward escape is likely to happen. If the viscosity is raised further, the tendency for the lubricant to escape shifts to the earlier stages of the reduction (See Figure 7), in accordance with the experimental results of Table $1^{7}$. 


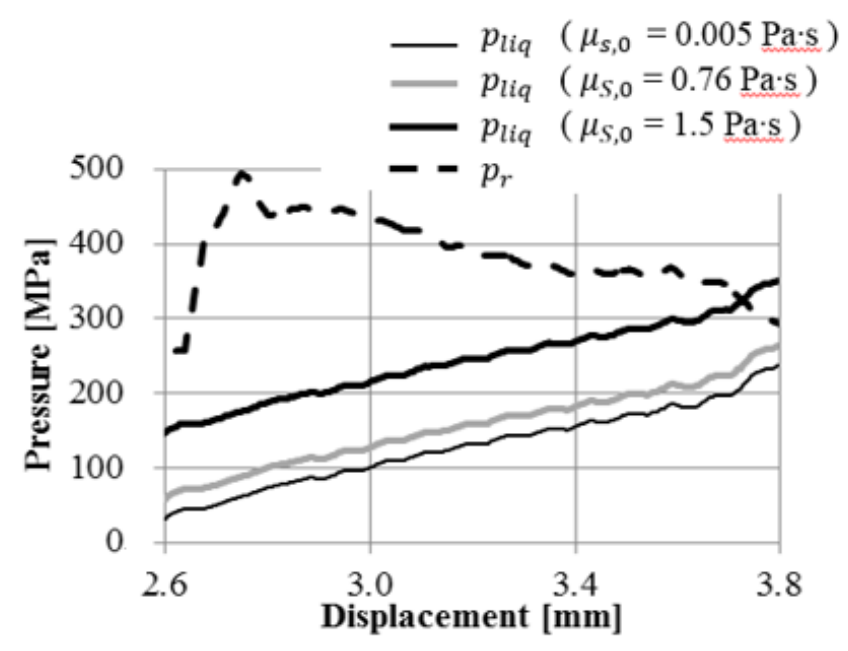

Figure 7. Evaluation of the liquid pressure $p_{\text {liq }}$ and the rear sealing pressure $p_{r}$ of the pocket with respect to displacement for various shear viscosity $\mu_{S, 0}$ values.

\section{MPHDL - Drawing speed}

The influence of the drawing speed on the backward lubricant escape was analysed by performing numerical simulations with three different drawing speeds $v=0.2,0.5,5 \mathrm{~mm} / \mathrm{s}$. Constant values of friction along the lower die $m=0$, lubricant viscosity $\mu_{S, 0}=0.15 \mathrm{~Pa} \cdot \mathrm{s}$ and back tension $\sigma_{b t}=0 \mathrm{MPa}$ were chosen in accordance with Table $1^{7}$.

Figure 8(a) shows that that liquid pressure $p_{\text {liq }}$ increases with the drawing speed $v$ while the rear sealing pressure $p_{r}$ has an opposite trend. This allows the two curves to intersect for drawing speeds of $v=0.5$ and $5.0 \mathrm{~mm} / \mathrm{s}$ in good agreement with the experimentally observed backward escape of lubricant summarized in Table $1^{7}$. In fact, the increase in drawing speed provides similar trends as the increase in shear viscosity because both situations raise the stress response of the lubricant.

Figure 8(b) illustrates the hydrostatic pressure in the lubricant and vertical stress distribution in the strip just before the backward escape occurs for drawing speed of $v=5 \mathrm{~mm} / \mathrm{s}$, which is also marked with a circle in Figure 8(a). The forward sealing pressure is high enough to restrict forward escape, whereas the backward sealing pressure is very close to the hydrostatic pressure in the lubricant. 


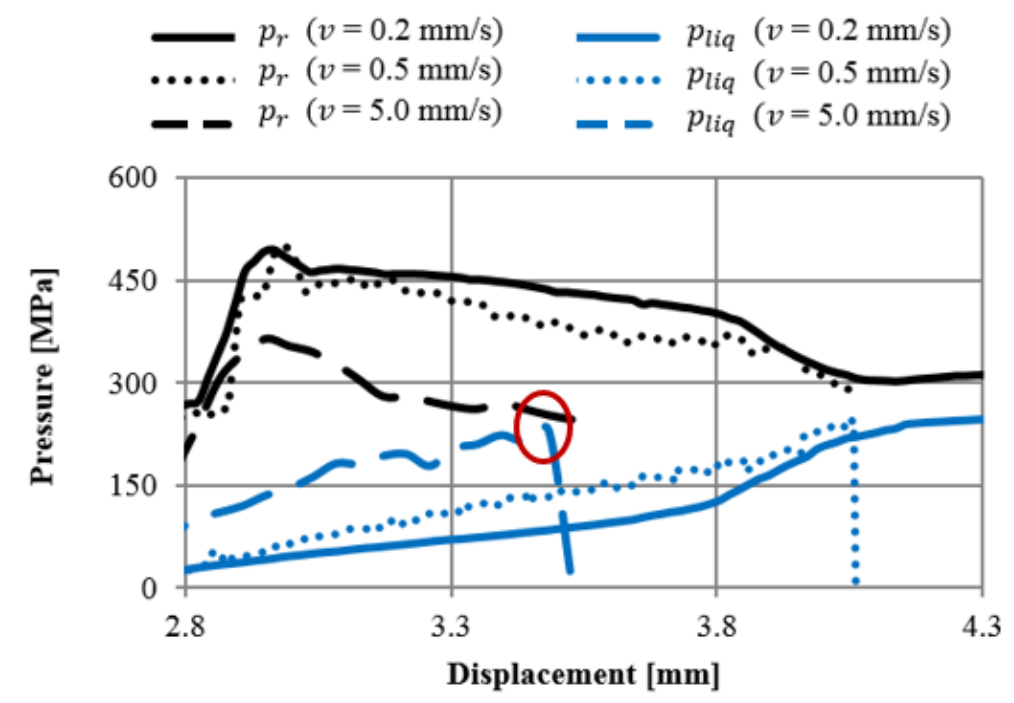

(a)

Pressure $[\mathrm{MPa}]$

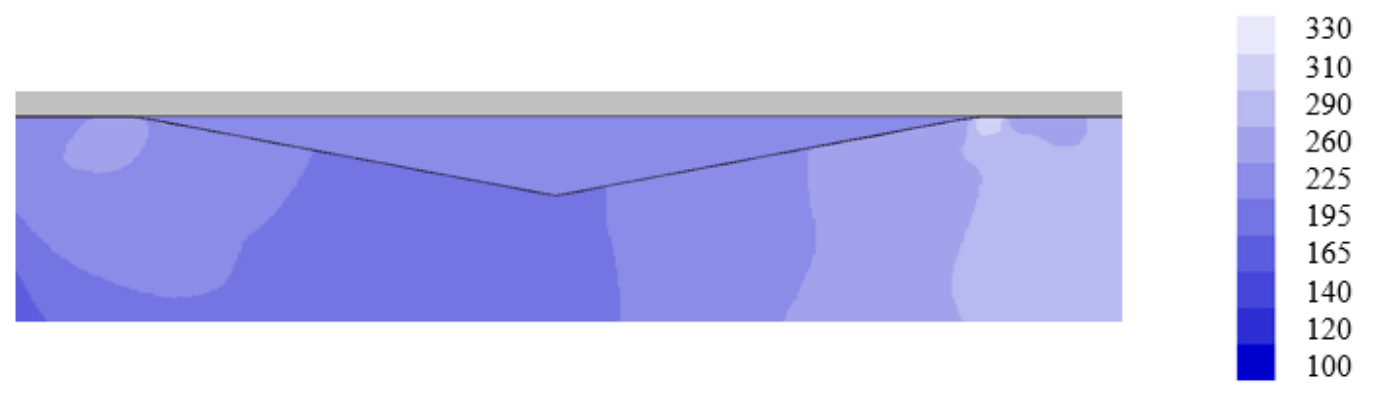

(b)

Figure 8. (a) Evolution of the liquid pressure $p_{\text {liq }}$ and rear sealing pressure $p_{r}$ of the pocket with displacement for different drawing speeds $v$ and (b) distribution of the vertical stress within the pocket and its surroundings just before the onset of escape corresponding to the location marked by the red circle in (a).

\section{Conclusions}

The finite element flow formulation can be utilized to simultaneously solve the plastic deformation of metals and the viscous flow of liquid lubricants. The required modifications to implement such a fullycoupled approach in existing computer programs are simple and mainly consist of replacing the stressstrain matrix built upon the constitutive equations of metals by the viscous, nearly incompressible, equations of Newtonian fluids. The velocity-pressure nature of the flow formulation ensures the required strong coupling, and the numerical difficulties caused by the heavy mesh distortions due to large 
differences between the stiffness of metals and liquids can be solved by an appropriate smoothing algorithm.

The new proposed approach was successfully applied to the drawing of a strip with a lubricant pocket in order to investigate the MPHSL and MPHDL mechanisms. Varying operative conditions for both MPHSL and MPHDL mechanisms were investigated by changing the main process parameters. The overall trends were characterized, and results compare well with experimental data and observations published by Bech et al. ${ }^{7}$.

Current limitations in the computer implementation of the proposed fully-coupled approach do not allow replicating the physical escape of the lubricant. Therefore, the approach is not capable of modelling experimental conditions having simultaneous forward and backward escapes of lubricant because it will only be able to determine the onset of the escape mode that will occur first and not what will happen afterwards, due to numerical difficulties arising from the mesh. This constraint requires further improvements in the future.

\section{Acknowledgments}

The work was supported by the Danish Council for Independent Research under grant no. DFF - 400500130. Paulo Martins would like to acknowledge Fundação para a Ciência e a Tecnologia de Portugal and IDMEC under LAETA - UID/EMS/50022/2013 and PDTC/EMS-TEC/0626/2014.

\section{References}

1. Kudo H. A note on the role of microscopically trapped lubricant at the tool-work interface. Int. J. Mech. Sci. 1965; 7: 383-388.

2. Nellemann T, Bay N, Wanheim T. Real area of contact and friction stress - the role of trapped lubricant. Wear, 1977; 43: 45-53.

3. Mizuno T, Okamoto M. Effect of lubricant viscosity at pressure and sliding velocity on lubricating conditions in the compression-friction test on sheet metals. J. of Lubr. Technol., 1982; 104: 53-59. 
4. Kudo H, Tsubouchi M, Takada H, Okamura K. An investigation into plasto-hydrodynamic lubrication with a cold sheet drawing test. Annu. CIRP, 1982; 31/1: 175-180.

5. Azushima A, Tsubouchi T, Kudo H., Direct observation of lubricant behaviors under the micro - PHL at the interface between workpiece and die. $3^{\text {rd }}$ International Conference on Technology of Plasticity, Kyoto, Japan, 1990, pp. 551-556.

6. Azushima A, Kudo H. Direct observation of contact behaviour to interpret the pressure dependence of the coefficient of friction in sheet metal forming. Annu. CIRP, 1995; 44/1: 209-212.

7. Bech J, Bay N, Eriksen M. Entrapment and escape of liquid lubricant in metal forming. Wear, 1999; 232: 134-139.

8. Shimizu I, Andreasen JL, Bech JI, Bay N. Influence of workpiece surface topography on the mechanisms of liquid lubrication in strip drawing. Trans. ASME, J. Tribol., 2001; 123: 290-294.

9. Lo S-W, Wilson WRD. A theoretical model of micro-pool lubrication in metal forming. Trans. ASME, J. Tribol., 1999; 121: 731-738.

10. Azushima A, Yanagida A, Tani S. Permeation of lubricant trapped within pocket into real contact area on the end surface of cylinder. Trans. ASME, J. Tribol., 2011; 133: 1-5.

11. Carretta Y, Boman R, Ponthot JP, Legrand N, Laugier M, Quang TN. Multiscale modelling of the micro-plasto-hydrodynamic lubrication - A crucial mechanism for friction in metal forming. 6th International Conference on Coupled Problems in Science and Engineering - Coupled Problems, Venice, Italy, 2015.

12. Zienkiewicz OC. Flow formulation for numerical solution of forming processes, in: Pittman FT, Zienkiewicz OC, Wood BD, Alexander JM (Eds.), Numerical Analysis of Forming Processes (John Wiley \& Sons: Chichester U.K., 1984.

13. Nielsen CV, Zhang W, Alves LM, Bay N, and Martins PAF. Modeling of Thermo-Electro-Mechanical Manufacturing Processes with Applications in Metal Forming and Resistance Welding. Series: SpringerBriefs in Applied Sciences and Technology. Subseries: SpringerBriefs in Manufacturing and Surface Engineering, 2013; ISBN 978-1-4471-4643-8.

14. Hartley P, Sturgess CEN, Rowe GW. Friction in finite-element analyses of metal forming processes. Int. J. Mech. Sci., 1979; 21: 301-311. 
15. Üstünyagiz E, Christiansen P, Nielsen CV, Bay N, Martins PAF. A fully-coupled approach for modelling plastic deformation and liquid lubrication in metal forming. $7^{\text {th }}$ International Conference on Tribology in Manufacturing Processes, Phuket, Thailand, 2016, pp. 45-56.

16. Nielsen CV, Fernandes JLM, Martins PAF. All-hexahedral meshing and remeshing for multi-object manufacturing applications. Computer Aided Design, 2013; 45: 911-922.

17. Üstünyagiz E, Christiansen P, Nielsen CV, Bay N, Martins PAF. A fully-coupled approach combining plastic deformation and liquid lubrication. $12^{\text {th }}$ International Conference on Numerical Methods in Industrial Forming Processes, Troyes, France, 2016. 